\title{
Jejunal pyogenic granuloma diagnosed and treated with spiral enteroscopy
}

A 67-year-old woman presented to our hospital with a 1-year history of melena. She also had history of hepatitis B and C virus-related liver cirrhosis complicated by hepatocellular carcinoma. On examination, her conjunctivae were pale and her nadir hemoglobin was $5.1 \mathrm{~g} / \mathrm{dL}$. Esophagogastroduodenoscopy and colonoscopy showed no definite bleeding source, and a diagnosis of obscure gastrointestinal bleeding was made. Capsule endoscopy demonstrated blood oozing in the proximal jejunum ( $\bullet$ Fig.1) and several angioectasia were identified in the small bowel. We performed spiral endoscopy perorally using an overtube (Discovery Small Bowel overtube; Spirus Medical, Stoughton, Massachusetts, USA) and an enteroscope (Fujinon EN-450T5; Fujinon, Saitama, Japan), which demonstrated a 1.5-cm sub-pedunculated tumor with superficial ulceration in the proximal jejunum, indicative of the bleeding point $(\bullet$ Fig. 2). We removed the tumor using snare polypectomy; bleeding from the tumor site after polypectomy was successfully stopped with hemoclips ( Fig.3). Pathological evaluation of the resected tumor revealed many capillaries of various sizes with acute and chronic inflammatory cell infiltrates, confirming the diagnosis of pyogenic granuloma ( $\mathbf{F i g . 4}$ ).

Pyogenic granuloma is a common lobular capillary hemangioma of the skin and mucosa and occurs with or without surface ulceration. Although rare, pyogenic granulomas can occur anywhere in the gastrointestinal tract with the small bowel accounting for $50 \%$ of all cases [1]. The tumor usually occurs in middle and late age, and it appears to have a higher prevalence in Asian populations [2]. Although the pathogenesis of pyogenic granuloma remains unknown, several etiological factors have been proposed, including infection, mechanical irritation, and hormones. Both the present case and our previously reported case had liver cirrhosis. Symptoms of small-bowel pyogenic granuloma include abdominal pain, hemorrhage, and

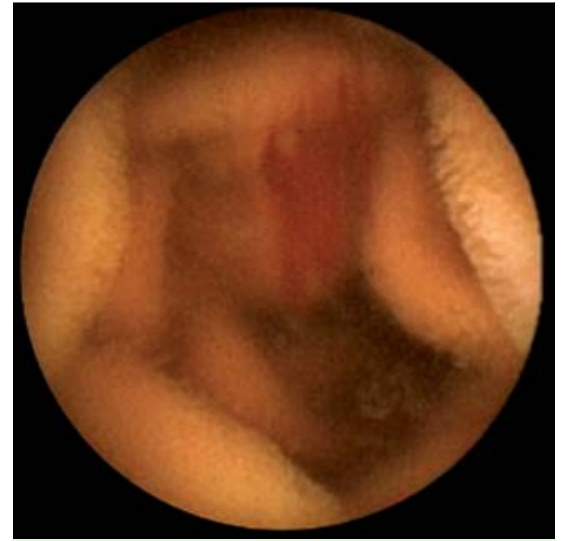

Fig. 1 Oozing blood identified in the proximal jejunum during capsule endoscopy in a 67year-old woman with a history of melena and liver cirrhosis.

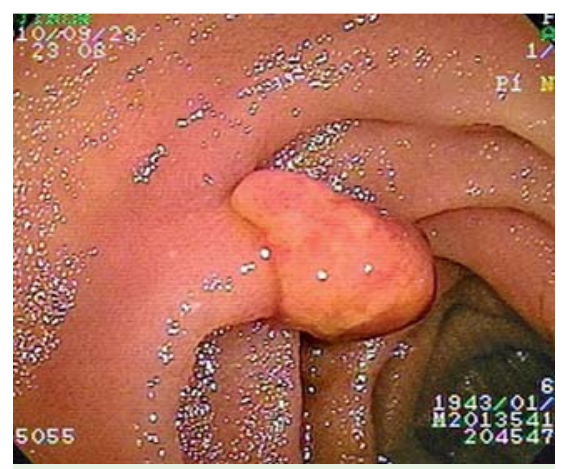

Fig. 2 Spiral enteroscopy showing a 1.5-cm sub-pedunculated tumor with surface ulceration.

intussusception. Capsule endoscopy and double-balloon enteroscopy are useful modalities in detecting small-bowel pyogenic granuloma $[3,4]$. Endoscopic resection via deep enteroscopy is an effective method of treating small-bowel pyogenic granulomas $[2,4,5]$, but it carries the risk of post-polypectomy bleeding owing to the tumor's rich blood supply and a high rate of recurrence if not resected completely.

Endoscopy_UCTN_Code_CCL_1AC_2AC

Competing interests: None

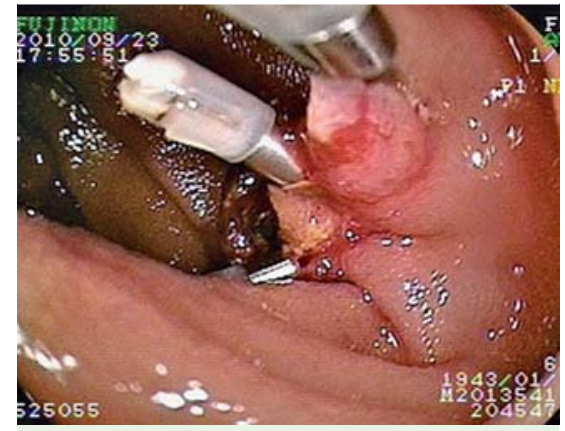

Fig. 3 The tumor bled easily after a snare polypectomy had been done; hemostasis was successfully achieved with three hemoclips.

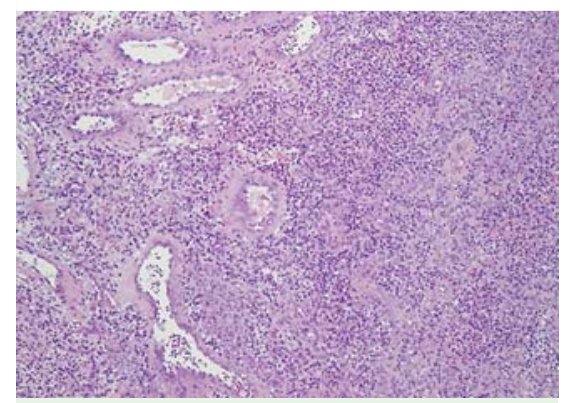

Fig. 4 Histological section of the resected tumor showing many capillaries of various sizes arranged in a lobular pattern, accompanied by acute and chronic inflammatory infiltrates and surface ulceration, confirming the diagnosis of pyogenic granuloma (hematoxylin and eosin, magnification $\times 100$ ).

\footnotetext{
J. W. Chou ${ }^{1,3}$, S. F. Chen ${ }^{1}$, C. Y. Yii', Y. S. Shih ${ }^{1}$, K. S. Cheng ${ }^{1,3}$, H. W. Chang ${ }^{2}$

${ }^{1}$ Division of Gastroenterology and Hepatology, Department of Internal Medicine, China Medical University Hospital, China Medical University, Taichung, Taiwan

2 Department of Pathology, China Medical University Hospital, China Medical University, Taichung, Taiwan

${ }^{3}$ College of Medicine, China Medical University, Taichung, Taiwan
} 


\section{References}

1 Moffatt DC, Warwryko P, Singh $H$. Pyogenic granuloma: an unusual cause of massive gastrointestinal bleeding from the small bowel. Can J Gastroenterol 2009; 23: 261 264

2 Park SY, Park CH, Lee WS et al. Pyogenic granuloma of the duodenum treated successfully by endoscopic mucosal resection. Gut Liver 2009; 3: 48-51

3 Chou JW, Lai HC, Lin YC. Image of the month. Pyogenic granuloma of the small bowel diagnosed by capsule endoscopy and double-balloon enteroscopy. Clin Gastroenterol Hepatol 2009; 7: A26

4 Shirakawa K, Nakamura T, Endo M et al. Pyogenic granuloma of the small intestine. Gastrointest Endosc 2007; 66: 827-828

5 Nagoya H, Tanaka S, Tatsuguchi A et al. Rare cause of obscure gastrointestinal bleeding due to pyogenic granuloma in the ileum detected by capsule endoscopy and treated with double balloon endoscopy. Dig Endosc 2010; 22 : $71-73$

\section{Bibliography}

Dol http://dx.doi.org/

10.1055/s-0031-1291425

Endoscopy 2012; 44: E2-E3

(C) Georg Thieme Verlag KG

Stuttgart · New York

ISSN 0013-726X

\section{Corresponding author}

\section{Dr K. S. Cheng}

Division of Gastroenterology and Hepatology Department of Internal Medicine China Medical University Hospital No. 2, Yuh-Der Road

North District

Taichung 40447

Taiwan

Fax: +886-4-22023119

codecol@yahoo.com.tw 